\title{
Evaluation of Adherence and Persistence Differences Between Adalimumab Citrate-Free and Citrate Formulations for Patients with Immune-Mediated Diseases in the United States
}

\author{
Martin Bergman · Pankaj Patel · Naijun Chen · Yonghua Jing • \\ Christopher D. Saffore
}

Received: October 19, 2020 / Accepted: November 7, 2020 / Published online: November 21, 2020

(C) The Author(s) 2020

\section{ABSTRACT}

Introduction: Our aim was to evaluate patient adherence and persistence with citrate-free adalimumab (ADA-CF), introduced in 2018 to reduce injection-site pain, compared with citrate-containing adalimumab (ADA-C).

Methods: This was a retrospective cohort study using a US claims database $\left(\mathrm{IBM}^{\circledR}\right.$ MarketScan $^{\circledR}$ Commercial and Medicare Supplemental Claims Database) from February 2018 to January 2020. Patients at least 18 years of age who were naïve to adalimumab 6 months before the index date (date of first adalimumab claim) and with at least 12 months of continuous medical and pharmacy coverage were eligible for the study. Adherence was assessed by determining the proportion of days covered (PDC) and the percentage of patients with $\mathrm{PDC} \geq 80 \%$ during the 12-month follow-up period. Persistence was evaluated by measuring the rate of discontinuation and days to discontinuation (i.e., time on

Electronic supplementary material The online version of this article (https://doi.org/10.1007/s40744020-00256-X) contains supplementary material, which is available to authorized users.

M. Bergman

Department of Medicine, Drexel University College

of Medicine, Philadelphia, PA, USA

P. Patel · N. Chen · Y. Jing · C. D. Saffore $(\bowtie)$

AbbVie Inc, North Chicago, IL, USA

e-mail: christopher.saffore@abbvie.com treatment) from the index date over the 12-month follow-up period. Continuous adherence outcomes (PDC) were evaluated using linear regression models. Binary adherence outcomes (PDC $\geq 80 \%$ ) were assessed using logistic regression models. Kaplan-Meier analysis and Cox proportional hazards models were used to assess persistence outcomes.

Results: There were 2195 and 1005 patients in the ADA-CF and ADA-C cohorts, respectively, with most using adalimumab for rheumatoid arthritis (ADA-CF 29.7\%, ADA-C 27.2\%) and psoriasis (ADA-CF $24.5 \%$, ADA-C 31.9\%). Significantly greater adherence was achieved with ADA-CF compared with ADA-C (mean PDC [standard deviation] 0.68 [0.30] vs 0.61 [0.32], $P<0.0001)$. A significantly greater percentage of patients receiving ADA-CF (47.2\%) vs ADA-C (39.6\%) had PDC $\geq 80 \%(P<0.0001)$. The discontinuation rate was significantly lower for the ADA-CF cohort (46.4\%) compared with ADA-C (55.9\%, $P<0.0001)$, resulting in a $27 \%$ lower likelihood of discontinuation during the 12-month follow-up period (hazard ratio 0.73; 95\% confidence interval $0.66,0.82 ; P<0.0001$ ) and longer time on treatment (260 vs 232 days, $P<0.0001)$.

Conclusion: Adherence and persistence are significantly improved with ADA-CF compared with ADA-C.

Keywords: Adalimumab; Adherence; Citrate; Citrate-free; Injection-site pain; Persistence 


\section{Key Summary Points}

Why carry out this study?

In 2018, citrate-free adalimumab was introduced to reduce injection-related pain.

Adherence and persistence were compared between citrate-free and citrate adalimumab.

\section{What was learned from this study?}

Significantly greater adherence was found with citrate-free vs citrate adalimumab.

Significantly better persistence was shown with citrate-free vs citrate adalimumab.

Reducing injection-site pain could potentially lead to better adherence and persistence.

\section{DIGITAL FEATURES}

This article is published with digital features to facilitate understanding of the article. You can access the digital features on the article's associated Figshare page. To view digital features for this article go to https://doi.org/10.6084/m9. figshare.13200950.

\section{INTRODUCTION}

Adalimumab is an anti-tumor necrosis factor (TNF) monoclonal antibody, initially approved in 2002, for the treatment of several immunerelated inflammatory diseases by subcutaneous injection [1]. The original formulation of adalimumab contains citrate (ADA-C), which is an inactive ingredient and is associated with injection-related pain [2-5]. One consequence of the use of citrate and its associated injectionsite pain is the potential of reduced adherence and persistence [6-8], which could lead to treatment failure and accelerated disease progression [9].

In August 2018, a citrate-free formulation of adalimumab (ADA-CF) was made available in the USA [1]. Additional changes made to the ADA-C formulation to reduce injection-site pain included reduction of the injection volume from $0.8 \mathrm{~mL}$ to $0.4 \mathrm{~mL}$ and the use of a smaller needle (29 vs 27 gauge) [10]. Injection volume and needle size and sharpness have been previously associated with injection-site pain $[5,11-13]$.

In a randomized crossover comparison of the ADA-CF and ADA-C formulations, there was clinically relevant and statistically significantly lower immediate injection-site pain among patients who received ADA-CF compared with those who received ADA-C [10]. Patients who received ADA-CF versus $\mathrm{ADA}-\mathrm{C}$ had a mean difference of -2.48 (95\% confidence interval $[\mathrm{CI}]-2.97$ to $-2.00 ; P<0.001)$ in patient-reported injection-related pain immediately after injection, as measured by visual analog scale of 0-10 cm [10]. ADA-CF was also shown to have a similar safety and tolerability profile compared with ADA-C [10].

Whether ADA-CF would lead to greater adherence and persistence compared with ADA-C is not known in clinical practice. Such information is important because of the consequences of poor adherence and persistence on patient outcomes, which include treatment failure and accelerated progression of disease [9]. In this study, we evaluated the adherence and persistence of adalimumab-naïve patients initiating either the ADA-CF or ADA-C formulations in clinical practice over a 1-year period using data from a US administrative claims database.

\section{METHODS}

\section{Study Design and Patients}

This was a retrospective cohort study using the IBM $^{\circledR}$ MarketScan ${ }^{\circledR}$ Commercial and Medicare Supplemental Claims Database for the period from February 2018 to January 2020. The database consists of data obtained from several 
million individuals annually, including employees and their spouses and dependents who are covered by employee-sponsored private health insurance and retirees covered by Medicare in the USA [14]. Employer-sponsored private health insurance plans included those from preferred provider, exclusive provider, and health maintenance organizations, as well as point-of-service, indemnity, and consumer-directed health plans [14]. Medical claims were linked to patient-level enrollment information and outpatient prescription drug claims [14]. Patient data were de-identified and met the Health Insurance Portability and Accountability Act of 1996 requirements to ensure patient anonymity [14].

Patients at least 18 years of age who were naïve to adalimumab within 6 months before the index date (first claim for adalimumab in August 2018 and beyond) with at least one claim based on a National Drug Code (Table 1 in the electronic supplementary material) for ADA-CF or ADA-C for the respective cohorts were eligible. Additional eligibility requirements included having continuous medical and pharmacy benefits for at least 6 months before and 12 months after the index date (12-month follow-up period). Patients were excluded if they had any claim for ADA-C (for those using ADA-CF) or ADA-CF (for those using ADA-C) in the 12-month follow-up period, had negative or missing days of supply for adalimumab, or had an adalimumab J-code claim in the 12 -month follow-up period.

\section{Outcomes}

Adherence was assessed by determining the proportion of days covered (PDC) and the percentage of patients with PDC $\geq 80 \%$ during the 12-month follow-up period in the ADA-CF and ADA-C cohorts. PDC was defined as the number of days covered by the index medication during the 12-month follow-up period divided by 365 days.

Persistence was evaluated by measuring the rate of discontinuation and time on treatment (i.e., days to discontinuation from the index date) in the 12-month follow-up period.
Discontinuation was defined as at least a 60-day continuous gap after fulfillment of days' supply in the previous adalimumab claim. A continuous gap of at least 60 days was chosen on the basis of a review of previous literature evaluating biologic treatment patterns in psoriatic arthritis [15-17].

\section{Statistical Methods}

Demographic and baseline characteristics measured in the 6-month period before the index date were summarized with descriptive statistics (mean, standard deviation for continuous endpoints, and $n$ [\%] for categoric endpoints). These characteristics included age, sex, Charlson comorbidity index, disease indication, payer type, and use of prior advanced therapies. Disease indications were identified using the International Classification of Diseases, Tenth Revision, Clinical Modification (ICD-10-CM) diagnosis codes (Table 2 in the electronic supplementary material) for immune-mediated diseases adalimumab is approved to treat (e.g., ankylosing spondylitis, Crohn's disease, hidradenitis suppurativa, plaque psoriasis, psoriatic arthritis, rheumatoid arthritis, ulcerative colitis, and uveitis). Linear regression models adjusted for baseline characteristics were used to compare continuous outcomes of PDC and time (days) on treatment between both cohorts. Logistic regression models adjusted for baseline characteristics were used to compare binary outcomes of adherence (percentage of patients with $\mathrm{PDC} \geq 80 \%$ ) and treatment discontinuation between both cohorts.

Persistence outcomes assessed as time to discontinuation were compared using KaplanMeier analysis, log-rank test, and Cox proportional hazards models adjusted for baseline characteristics. Hazard ratios and 95\% CIs generated from the Cox model were estimated for the likelihood of treatment discontinuation. A two-sided alpha error level of 0.05 was used to indicate statistical significance in all analyses. Sample selection and creation of analytic variables were performed using the Instant Health Data platform (Panalgo, Boston, MA, USA). 


\section{RESULTS}

\section{Patients}

There were 2195 patients in the ADA-CF cohort and 1005 patients in the ADA-C cohort who met the study criteria (Fig. 1). Baseline characteristics between the two cohorts were similar, including the proportion of patients receiving prior advanced therapies (Table 1). The greatest proportion of patients using either formulation of adalimumab was for the treatment of rheumatoid arthritis (ADA-CF 29.7\%, ADA-C 27.2\%) and psoriasis (ADA-CF 24.5\%, ADA-C 31.9\%) (Table 1).

\section{Adherence for Patients Receiving ADA-CF Versus ADA-C}

Patients who received ADA-CF had significantly greater adherence during the 12-month followup period compared with those who received ADA-C, with a mean (standard deviation) PDC of 0.68 (0.30) compared with $0.61 \quad(0.32)$
$(P<0.0001) \quad$ (Fig. 2a). The percentage of patients with a PDC $\geq 80 \%$ was significantly greater by $19.2 \%$ with $\mathrm{ADA}-\mathrm{CF}$ compared with ADA-C (47.2\% vs $39.6 \%, P<0.0001)$. (Fig. $2 b)$.

\section{Persistence for Patients Receiving ADA-CF Versus ADA-C}

Persistence on adalimumab was significantly better for patients who received ADA-CF versus ADA-C based on the 12-month discontinuation rate and time on treatment (Fig. 3). The discontinuation rate was significantly lower by $17 \%$ in the ADA-CF cohort compared with ADA-C (46.4\% vs $55.9 \%, P<0.0001)$ (Figs. $3 \mathrm{a}$ and 4 ). This difference resulted in a $27 \%$ lower likelihood that a patient would discontinue treatment during the 12-month follow-up period for the ADA-CF cohort versus the ADA-C cohort (hazard ratio 0.73; 95\% CI 0.66, 0.82; $P<0.0001$ ). Consequently, patients who received ADA-CF were on treatment for a significantly longer time ( 28 days) than patients who received ADA-C (260 vs 232 days,

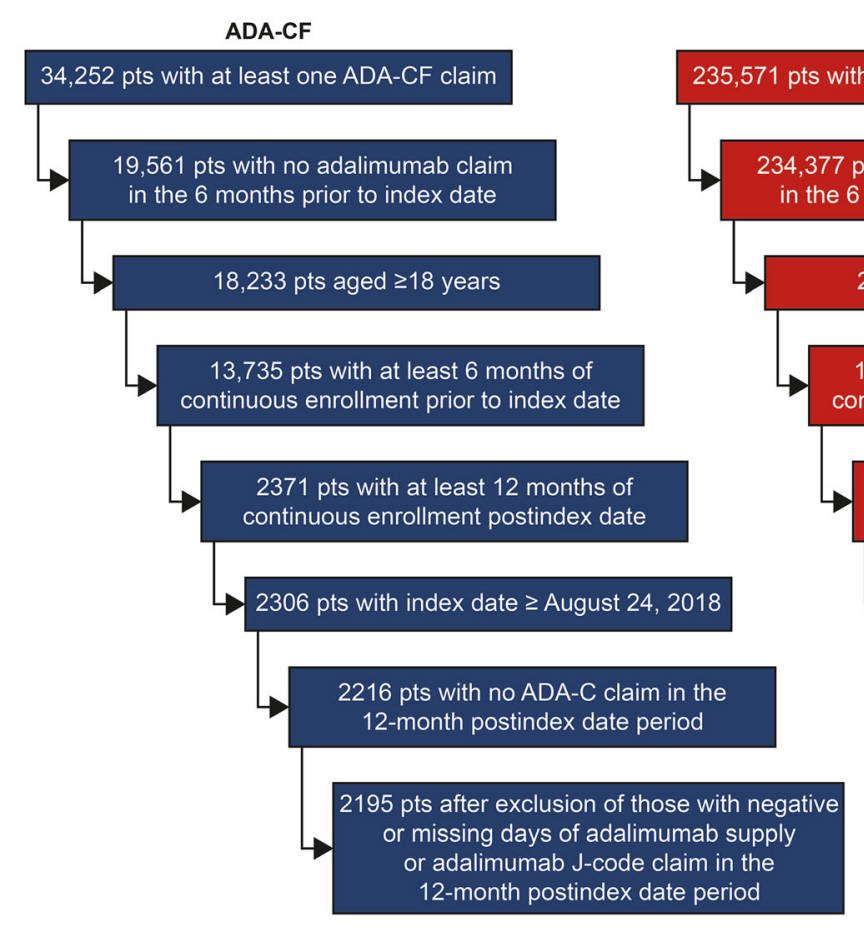

ADA-C

$23,637 \mathrm{pts}$ with at least 6 months of continuous enrollment prior to index date

87,048 pts with at least 12 months of continuous enrollment postindex date

1633 pts with index date $\geq$ August 24, 2018

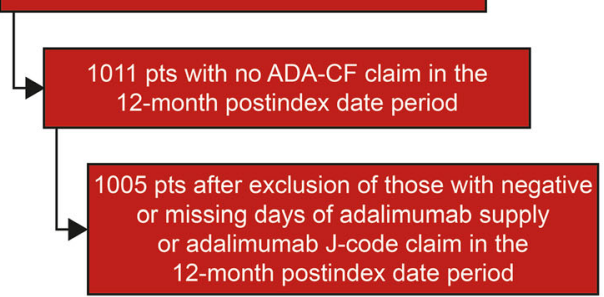

Fig. 1 Study population selection. ADA-C adalimumab citrate formulation, ADA-CF adalimumab citrate-free formulation, pts patients 
Table 1 Baseline demographics and clinical characteristics

\begin{tabular}{lll}
\hline & Adalimumab citrate-free $(\boldsymbol{N}=\mathbf{2 1 9 5})$ & Adalimumab citrate $(\boldsymbol{N}=\mathbf{1 0 0 5})$ \\
\hline $\begin{array}{l}\text { Age, mean (SD), years } \\
\text { Sex, } n \text { (\%) }\end{array}$ & $46.0(13.1)$ & $46.0(12.9)$ \\
Female & $1361(62.0)$ & $571(56.8)$ \\
Male & $834(38.0)$ & $434(43.2)$ \\
Charlson comorbidity index, mean (SD) & $0.62(0.88)$ & $0.65(0.99)$ \\
Payer, $n$ (\%) & & \\
Commercial & $2129(97.0)$ & $974(96.9)$ \\
Medicare & $66(3.0)$ & $31(3.1)$ \\
Disease indication, $n(\%)^{\mathrm{a}}$ & & $53(5.3)$ \\
Ankylosing spondylitis & $143(6.5)$ & $128(12.7)$ \\
Crohn's disease & $332(15.1)$ & $66(6.6)$ \\
Hidradenitis suppurativa & $125(5.7)$ & $321(31.9)$ \\
Psoriasis & $538(24.5)$ & $155(15.4)$ \\
Psoriatic arthritis & $411(18.7)$ & $273(27.2)$ \\
Rheumatoid arthritis & $653(29.7)$ & $107(10.6)$ \\
Ulcerative colitis & $255(11.6)$ & $43(4.3)$ \\
Uveitis & $84(3.8)$ & $206(20.5)$ \\
Prior advanced therapies, $n(\%)^{\mathrm{a}, \mathrm{b}}$ & $480(21.9)$ & \\
\hline
\end{tabular}

$S D$ standard deviation

a $\geq 1$ claim within 6 months before the index date

b Includes abatacept, anakinra, apremilast, baricitinib, brodalumab, certolizumab pegol, etanercept, golimumab, guselkumab, infliximab, ixekizumab, natalizumab, risankizumab-rzaa, rituximab, sarilumab, secukinumab, tildrakizumab-asmn, tocilizumab, tofacitinib, upadacitinib, ustekinumab, and vedolizumab

$P<0.0001$ ) (Fig. 3b) during the 12-month follow-up period.

\section{DISCUSSION}

In this study, we evaluated whether ADA-CF, a new formulation of adalimumab developed to reduce injection-site pain, was associated with greater adherence and persistence compared with ADA-C in clinical practice. To the best of our knowledge, this is the first study evaluating patient adherence and persistence patterns between those receiving ADA-CF versus ADA-C.
We determined that patients who received ADA-CF had significantly greater adherence compared with ADA-C, with a $19.2 \%$ higher percentage of patients with a $\mathrm{PDC} \geq 80 \%$. Persistence was also better for patients who received ADA-CF compared with ADA-C, with patients having a $17 \%$ lower discontinuation rate and a greater length of time on the medication. The adherence and persistence results with ADA-CF versus ADA-C were also consistent across rheumatology (i.e., rheumatoid arthritis, psoriatic arthritis, ankylosing spondylitis), dermatology (i.e., psoriasis, hidradenitis suppurativa), and gastroenterology (i.e., ulcerative 


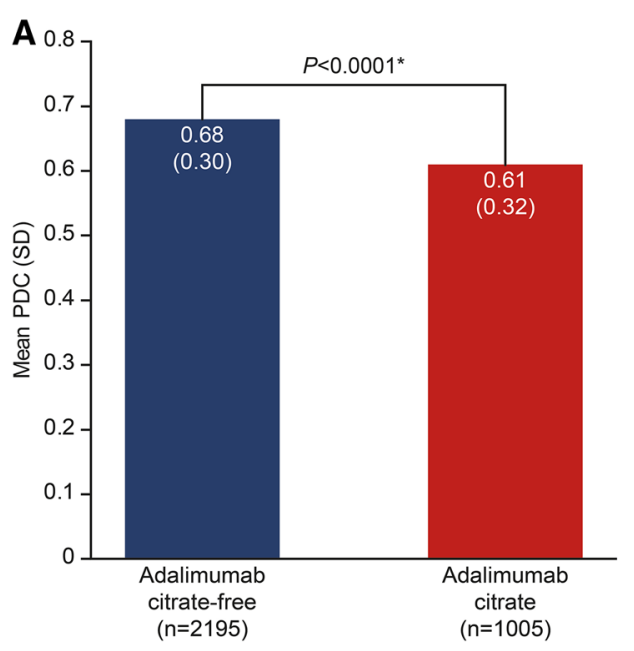

Fig. 2 Adherence of patients receiving adalimumab citrate-free versus citrate formulations. a Mean PDC. b Percentage of patients with $\mathrm{PDC} \geq 80 \%$. ${ }^{*} P<0.0001$ based on linear regression model (PDC) and logistic regression model (PDC $\geq 80 \%)$. Linear and logistic

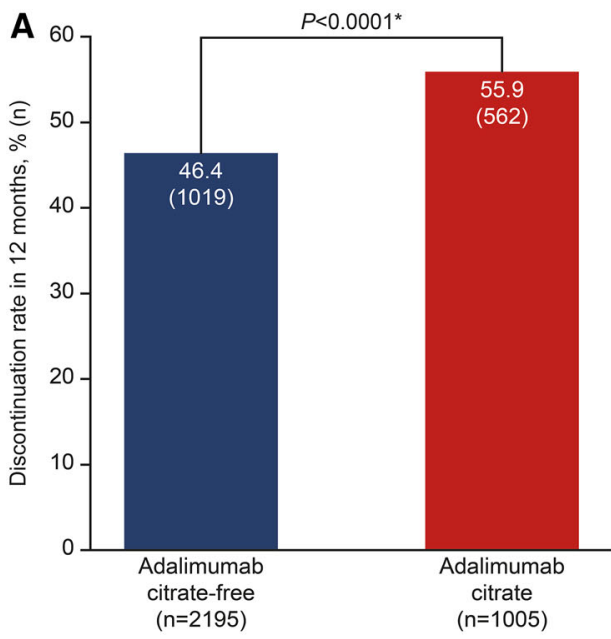

Fig. 3 Rate of discontinuation and time on treatment for patients receiving adalimumab citrate-free versus citrate. a Discontinuation rate. $\mathbf{b}$ Time on treatment. ${ }^{*} P<0.0001$ based on logistic regression model (discontinuation rate)

colitis, Crohn's disease) disease indications (data not shown).

Nonadherence to medications, particularly injectable treatments, is a major issue that can lead to poorer health outcomes, increased mortality, and greater healthcare resource

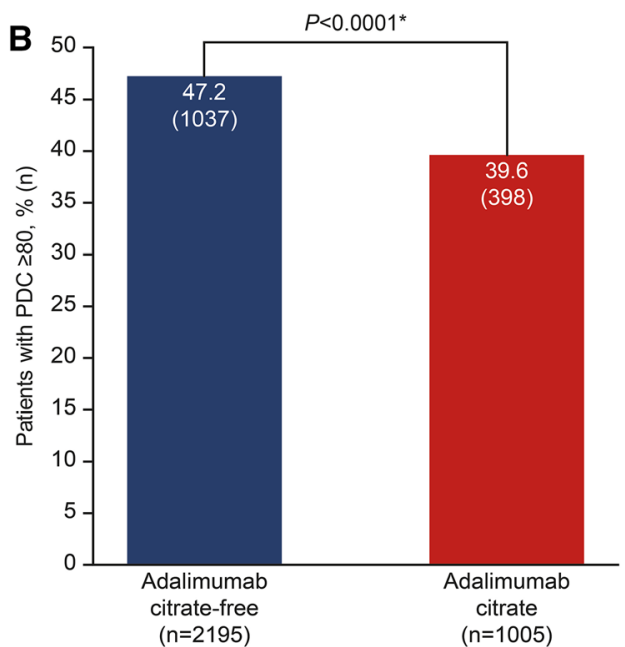

regression models adjusted for age, sex, Charlson comorbidity index, disease indication, payer type, and biologicnaïve status. PDC proportion of days covered, SD standard deviation

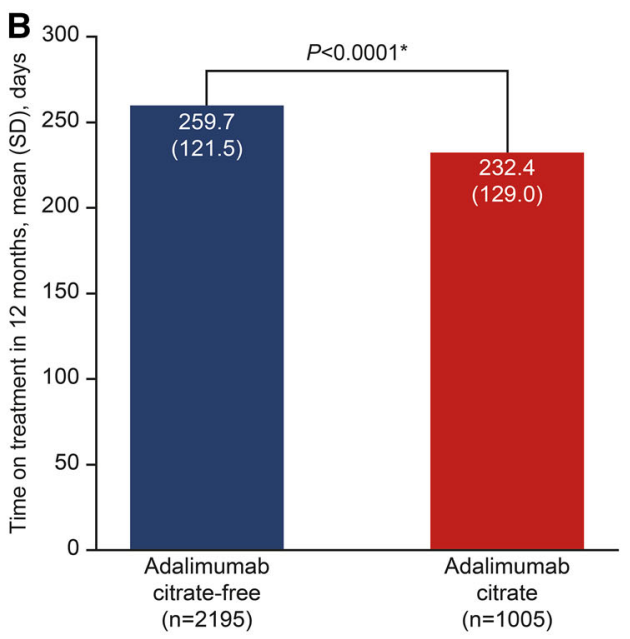

and linear regression model (time on treatment). Linear and logistic regression models adjusted for age, sex, Charlson comorbidity index, disease indication, payer type, and biologic-naïve status. SD standard deviation

utilization and costs [18-21]. Therefore, developing formulations that can lead to greater adherence and persistence with injectable treatments is highly important for improving patient outcomes. This point is particularly relevant for anti-TNF therapies, for which 


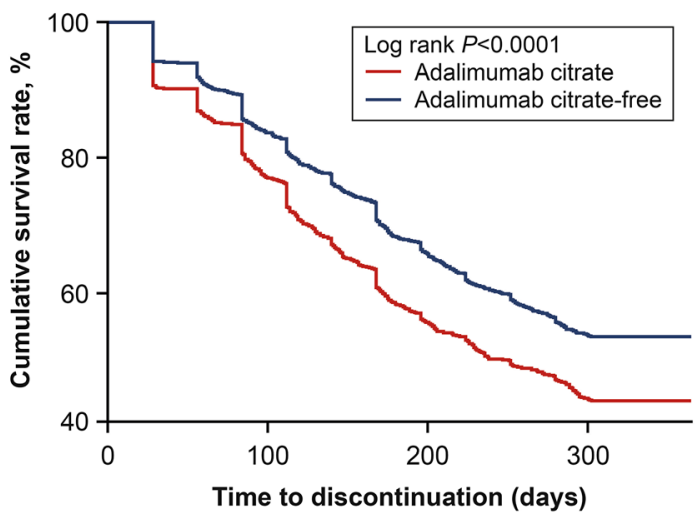

Fig. 4 Survival analysis of the time to discontinuation with adalimumab citrate-free versus adalimumab citrate formulations

nonadherence rates are greater than $40 \%$ [22-24]. Consequently, there is a critical need to develop strategies to promote patient adherence for all injectables, including anti-TNF therapies.

One such strategy being employed by AbbVie, the manufacturer of adalimumab, is a patient support program (PSP) that is being provided free of charge to patients receiving adalimumab [22]. The PSP assists patients with medication costs, nurse support, injection training, medication training, and pen disposal [22]. In a longitudinal, retrospective cohort study of 2386 patients performed prior to the introduction of ADA-CF, patients who received ADA-C and were in the PSP $(n=1199)$ had $14 \%$ greater adherence in the 12-month follow-up period compared with patients not in the PSP ( $n=1187,67.0 \%$ vs $58.8 \%, P<0.001)$ [22]. Additionally, the discontinuation rate was $14 \%$ lower for the patients in the PSP compared with those not in the PSP $(39.7 \%$ vs $46.2 \%$, $P=0.001$ ) [22]. These findings suggest the PSP could provide additional improvements in adherence and persistence for patients receiving ADA-CF in the PSP compared with those not in the PSP.

One important factor that influences patient adherence and persistence is injection-site pain [6-8], an adverse event that occurs in $12 \%$ of patients who receive ADA-C [25]. In a survey of patients receiving subcutaneous biologic therapy for rheumatoid arthritis, $41 \%$ of patients discontinued treatment at least in part because of injection experience, which included approximately $28 \%$ who reported injection-site pain, burning, or discomfort during or after the injection [6]. In an interview of healthcare professionals, patient perception of pain and needle size were among the highest perceived barriers to adherence, with $39 \%$ and $28 \%$ of respondents, respectively, naming these factors for subcutaneous injections [7]. Another study that evaluated the influence of factors on antiTNF treatment adherence found that lower injection-site pain and skin perceptions were significantly associated with the increased odds of medication adherence $(P=0.0008)$ [8]. The results from this current study support the concept that by reducing injection-site pain, one can significantly improve patient adherence and persistence.

A strength of this study is the use of a large patient population from the IBM ${ }^{\circledR}$ MarketScan ${ }^{\circledR}$ Commercial and Medicare Supplemental Claims Database to adequately evaluate the adherence and persistence of patients receiving different formulations of adalimumab. As this is a payer claims database, a limitation of this study was that there was no confirmation that patients who were prescribed adalimumab actually used the medication. Also, prescriptions for adalimumab filled outside the claims database were not captured. Additionally, reasons for discontinuation were not collected in the claims database and therefore were not available. Furthermore, the generalizability of these results is limited to patient populations with either commercial insurance or Medicare. Additional factors that could influence adherence and persistence, such as clinician or patient preference for a given formulation or patient enrollment in a PSP and clinical response to treatment, could not be captured or evaluated.

\section{CONCLUSION}

Patients who received ADA-CF had significantly greater adherence, fewer discontinuations, and longer time on treatment compared with those who received ADA-C. As improved adherence and persistence are related to decreased 
treatment failure and disease progression rates [18-20], ADA-CF should be considered by clinicians over ADA-C when managing patients with immune-mediated diseases. It will be of interest in a future study to determine whether such improvements lead to reduced treatment failure and lower disease progression rates for patients being treated with ADA-CF versus ADA-C.

\section{ACKNOWLEDGEMENTS}

Funding. This work/study was funded by AbbVie Inc. AbbVie participated in the study design, research, data collection, analysis and interpretation of data, writing, reviewing, and approving the publication. All authors had access to the data results, and participated in the development, review, and approval of this publication. The journal's Rapid Service Fee was funded by AbbVie.

Authorship. All named authors meet the International Committee of Medical Journal Editors (ICMJE) criteria for authorship for this article, take responsibility for the integrity of the work as a whole, and have given their approval for this version to be published.

Medical Writing and Editorial Assistance. Medical writing services were provided by Alan Saltzman, PhD, CMPP, of JK Associates, Inc., (a member of the Fishawack Group of Companies), Conshohocken, PA; this support was funded by AbbVie Inc.

Disclosures. Martin Bergman is a consultant/speaker for AbbVie, Amgen, BMS, Genentech, Gilead, Janssen, Merck, Novartis, Pfizer, Regeneron, Sanofi, and Sandoz and is a shareholder of JNJ (parent of Janssen). Pankaj Patel, Naijun Chen, Yonghua Jing, and Christopher D. Saffore are employees and stockholders of AbbVie.

Compliance with Ethics Guidelines. Patient data were de-identified and met the Health Insurance Portability and Accountability Act of
1996 requirements to ensure patient anonymity [14].

Data Availability. The datasets generated during and/or analyzed during the current study are available from the corresponding author on reasonable request.

Open Access. This article is licensed under a Creative Commons Attribution-NonCommercial 4.0 International License, which permits any non-commercial use, sharing, adaptation, distribution and reproduction in any medium or format, as long as you give appropriate credit to the original author(s) and the source, provide a link to the Creative Commons licence, and indicate if changes were made. The images or other third party material in this article are included in the article's Creative Commons licence, unless indicated otherwise in a credit line to the material. If material is not included in the article's Creative Commons licence and your intended use is not permitted by statutory regulation or exceeds the permitted use, you will need to obtain permission directly from the copyright holder. To view a copy of this licence, visit http://creativecommons.org/licenses/by$\mathrm{nc} / 4.0 /$.

\section{REFERENCES}

1. Humira ${ }^{\circledR}$ prescribing information. AbbVie Inc. North Chicago, IL USA. 2020. https://www. rxabbvie.com/pdf/humira.pdf. Accessed 16 Jun 2020.

2. Frenken LA, van Lier HJ, Jordans JG, et al. Identification of the component part in an epoetin alfa preparation that causes pain after subcutaneous injection. Am J Kidney Dis. 1993;22:553-6. https:// doi.org/10.1016/s0272-6386(12)80928-0.

3. Laursen T, Hansen B, Fisker S. Pain perception after subcutaneous injections of media containing different buffers. Basic Clin Pharmacol Toxicol. 2006;98:218-21. https://doi.org/10.1111/j.17427843.2006.pto_271.x.

4. Veys N, Dhondt A, Lameire N. Pain at the injection site of subcutaneously administered erythropoietin: phosphate-buffered epoetin alpha compared to 
citrate-buffered epoetin alpha and epoetin beta. Clin Nephrol. 1998;49:41-4.

5. Usach I, Martinez R, Festini T, Peris JE. Subcutaneous injection of drugs: literature review of factors influencing pain sensation at the injection site. Adv Ther. 2019;36:2986-96. https://doi.org/10.1007/ s12325-019-01101-6.

6. Bolge SC, Goren A, Tandon N. Reasons for discontinuation of subcutaneous biologic therapy in the treatment of rheumatoid arthritis: a patient perspective. Patient Prefer Adherence. 2015;9:121-31. https://doi.org/10.2147/PPA.S70834.

7. Gandell DL, Bienen EJ, Gudeman J. Mode of injection and treatment adherence: results of a survey characterizing the perspectives of health care providers and US women 18-45 years old. Patient Prefer Adherence. 2019;13:351-61. https://doi.org/10. 2147/PPA.S187120.

8. Salaffi F, Di Carlo M, Farah S, Carotti M. Adherence to subcutaneous anti-TNF-alpha agents in patients with rheumatoid arthritis is largely influenced by pain and skin sensations at the injection site. Int $\mathrm{J}$ Rheum Dis. 2020;23:480-7. https://doi.org/10. 1111/1756-185X.13803.

9. Grijalva CG, Chung CP, Arbogast PG, Stein CM, Mitchel EF Jr, Griffin MR. Assessment of adherence to and persistence on disease-modifying antirheumatic drugs (DMARDs) in patients with rheumatoid arthritis. Med Care. 2007;45:S66-76. https://doi. org/10.1097/MLR.0b013e318041384c.

10. Nash P, Vanhoof J, Hall S, et al. Randomized crossover comparison of injection site pain with 40 $\mathrm{mg} / 0.4$ or $0.8 \mathrm{~mL}$ formulations of adalimumab in patients with rheumatoid arthritis. Rheumatol Ther. 2016;3:257-70. https://doi.org/10.1007/ s40744-016-0041-3.

11. Heise $\mathrm{T}$, Nosek $\mathrm{L}$, Dellweg $\mathrm{S}$, et al. Impact of injection speed and volume on perceived pain during subcutaneous injections into the abdomen and thigh: a single-centre, randomized controlled trial. Diabetes Obes Metab. 2014;16:971-6. https://doi. org/10.1111/dom.12304.

12. Anderson G, Meyer D, Herrman CE, et al. Tolerability and safety of novel half milliliter formulation of glatiramer acetate for subcutaneous injection: an open-label, multicenter, randomized comparative study. J Neurol. 2010;257:1917-23. https://doi.org/ 10.1007/s00415-010-5779-x.

13. Jaber A, Bozzato GB, Vedrine L, Prais WA, Berube J, Laurent PE. A novel needle for subcutaneous injection of interferon beta-1a: effect on pain in volunteers and satisfaction in patients with multiple sclerosis. BMC Neurol. 2008;8:38. https:// doi.org/10.1186/1471-2377-8-38.

14. IBM Watson Health. White paper. IBM MarketScan Research Databases for life sciences researchers 2018. https://www.ibm.com/downloads/cas/ ONKLE57Y. Accessed 17 June 2020.

15. Chastek B, Fox KM, Watson C, Gandra SR. Etanercept and adalimumab treatment patterns in psoriatic arthritis patients enrolled in a commercial health plan. Adv Ther. 2012;29:691-7. https://doi. org/10.1007/s12325-012-0039-3.

16. Bonafede M, Johnson BH, Fox KM, Watson C, Gandra SR. Treatment patterns with etanercept and adalimumab for psoriatic diseases in a real-world setting. J Dermatolog Treat. 2013;24:369-73. https://doi.org/10.3109/09546634.2012.755255.

17. Wu JJ, Pelletier C, Ung B, Tian M. Treatment patterns and healthcare costs among biologic-naive patients initiating apremilast or biologics for the treatment of psoriatic arthritis: results from a US claims analysis. Curr Med Res Opin. 2020;36: 169-76. https://doi.org/10.1080/03007995.2019. 1668204 .

18. World Health Organization. Adherence to longterm therapies: evidence for action. 2003. http:// www.who.int/chp/knowledge/publications/ adherence_full_report.pdf. Accessed 16 Jun 2020.

19. Simpson SH, Eurich DT, Majumdar SR, et al. A meta-analysis of the association between adherence to drug therapy and mortality. BMJ. 2006;333:15. https://doi.org/10.1136/bmj.38875.675486.55.

20. Bluett J, Morgan C, Thurston L, et al. Impact of inadequate adherence on response to subcutaneously administered anti-tumour necrosis factor drugs: results from the Biologics in Rheumatoid Arthritis Genetics and Genomics Study Syndicate cohort. Rheumatology (Oxford). 2015;54:494-9. https://doi.org/10.1093/rheumatology/keu358.

21. Hughes DA, Bagust A, Haycox A, Walley T. The impact of non-compliance on the cost-effectiveness of pharmaceuticals: a review of the literature. Health Econ. 2001;10:601-15. https://doi.org/10. 1002/hec.609.

22. Rubin DT, Mittal M, Davis M, Johnson S, Chao J, Skup M. Impact of a patient support program on patient adherence to adalimumab and direct medical costs in Crohn's disease, ulcerative colitis, rheumatoid arthritis, psoriasis, psoriatic arthritis, and ankylosing spondylitis. J Manag Care Spec Pharm. 2017;23:859-67. https://doi.org/10.18553/ jmcp.2017.16272. 
23. Borah BJ, Huang X, Zarotsky V, Globe D. Trends in RA patients' adherence to subcutaneous anti-TNF therapies and costs. Curr Med Res Opin. 2009;25: 1365-77. https://doi.org/10.1185/ 03007990902896386.

24. Yazici Y, Krasnokutsky S, Barnes JP, Hines PL, Wang J, Rosenblatt L. Changing patterns of tumor necrosis factor inhibitor use in 9074 patients with rheumatoid arthritis. J Rheumatol. 2009;36:907-13. https://doi.org/10.3899/jrheum.080592.

25. Mease PJ. Adalimumab in the treatment of arthritis. Ther Clin Risk Manag. 2007;3:133-48. https://doi. org/10.2147/tcrm.2007.3.1.133. 